\title{
Longitudinal phase space reconstruction for a heavy ion accelerator
}

\author{
S. Lauber, ${ }^{*}$ K. Aulenbacher, W. Barth $\odot$, F. Dziuba, and J. List \\ Helmholtz Institute Mainz, Staudingerweg 18, D-55128 Mainz, Germany; \\ GSI Helmholtzzentrum für Schwerionenforschung GmbH, Planckstraße 1, D-64291 Darmstadt, Germany; \\ and Johannes Gutenberg University, Mainz, Germany \\ C. Burandt, V. Gettmann, T. Kürzeder, M. Miski-Oglu, P. Forck, M. Heilmann, A. Rubin, \\ T. Sieber, and S. Yaramyshev \\ GSI Helmholtzzentrum für Schwerionenforschung, Planckstraße 1, D-64291 Darmstadt, Germany \\ H. Podlech@ and M. Schwarz $\odot$ \\ Goethe University, Institute for Applied Physics, \\ Max-von-Laue-Straße 1, D-60438 Frankfurt a. M., Germany
}

(Received 19 February 2020; accepted 19 October 2020; published 13 November 2020)

\begin{abstract}
At the GSI Helmholtzzentrum für Schwerionenforschung (GSI) in Darmstadt, Germany, a prototype cryomodule (advanced demonstrator) for the superconducting (SC) continuous wave (CW) Helmholtz Linear Accelerator (HELIAC) is under construction. A transport line, comprising quadrupole lenses, rebuncher cavities, beam correctors, and adequate beam instrumentation has been built to deliver the beam from the GSI 1.4 MeV/u High Charge Injector (HLI) to the advanced demonstrator, which offers a test environment for SC CW multigap cavities. In order to achieve proper phase space matching, the beam from the HLI must be characterized in detail. In a dedicated machine experiment the bunch shape has been measured with a nondestructive bunch shape monitor (BSM). Therefore, different bunch projections were obtained by altering the voltage of two rebunchers. These measurements were combined with dedicated beam dynamics simulations using the particle tracking code DYNAMION. The longitudinal bunch shape and density distribution at the beginning of the matching line are fully characterized by a tomographic reconstruction method based on a non-negative least square minimization approach.
\end{abstract}

DOI: 10.1103/PhysRevAccelBeams.23.114201

\section{INTRODUCTION}

Super heavy element (SHE) research performs particle collision experiments with medium weight to heavy ions on heavy targets to cause fusion evaporation reactions. Extremely small cross-sections make reliable long-term operation crucial for the experiments [1,2]. While the GSI Universal Linear Accelerator (UNILAC) [3-7] is upgraded as an exclusive injector for the Facility for Antiproton and Ion Research (FAIR) $[3,7,8]$, a new SC CW heavy ion linear accelerator is built at GSI to keep the SHE research competitive [9]. This project is carried out by GSI and Helmholtz Institute Mainz (HIM) [10,11] under key support of the Goethe University Frankfurt (GUF) $[12,13]$ and in collaboration with the Moscow Engineering Physics

\footnotetext{
*s.lauber@gsi.de

Published by the American Physical Society under the terms of the Creative Commons Attribution 4.0 International license. Further distribution of this work must maintain attribution to the author(s) and the published article's title, journal citation, and DOI.
}

Institute (MEPhI) and the Moscow Institute for Theoretical and Experimental Physics (KI-ITEP) [14-16]. For different modern facilities worldwide, the operation of SC and CWLinacs is a key technology, as for the Spallation Neutron Source (SNS) in the U.S. [17], or medium energy applications in isotope generation, material science and boronneutron capture therapy [18-20]. All these ambitious projects strongly rely on proper beam diagnostics, as minimal beam loss is a critical requirement to the machines as well as for superconducting multigap cavities [21,22].

\section{A. Helmholtz linear accelerator}

In the future, a new warm injector has to provide a $1.4 \mathrm{MeV} / \mathrm{u} \mathrm{CW}$ heavy ion beam for the SC HELIAC [23]. It comprises of a radio frequency quadrupole (RFQ) and an interdigital $\mathrm{H}$-mode cavity (IH) together with two rebuncher cavities. Four cryomodules with compact $\mathrm{SC} \mathrm{CH}$ cavities [12,24-26], SC solenoids and SC rebunchers [13] form the SC HELIAC section. The main features of the accelerator are a variable output energy (see Table I), the capability to provide for $\mathrm{CW}$ operation and a low momentum spread [27]. The accurate implementation 
TABLE I. HELIAC design specifications [23].

\begin{tabular}{lc}
\hline \hline & Value \\
\hline Mass/charge & $\leq 6$ \\
Frequency & $216.816 \mathrm{MHz}$ \\
Mean beam current & $1 \mathrm{~mA}$ \\
Injection energy & $1.4 \mathrm{MeV} / \mathrm{u}$ \\
Variable output energy & $3.5 \mathrm{MeV} / \mathrm{u} \mathrm{to} 7.3 \mathrm{MeV} / \mathrm{u}$ \\
Output energy spread & $\pm 3 \mathrm{keV} / \mathrm{u}$ \\
Repetition rate & continuous wave \\
Temperature & $4 \mathrm{~K}$ \\
\hline \hline
\end{tabular}

of the beam dynamics design [28] is essential for the $\mathrm{CW}$-Linac project, as beam losses must be minimized to avoid the degradation of the superconducting cavities. Therefore, robust beam diagnostic methods are required, for the commissioning and routine operation [29,30]. The HELIAC stays in line with diverse ambitious linac projects at GSI [31,32], namely the FAIR proton linac [33], the UNILAC proton beam delivery [34-36], the linear heavy ion decelerator HITRAP (Heavy Ion TRAP) [37] and the LIGHT (Laser Ion Generation, Handling and Transport) facility for laser acceleration of protons and heavy ions [38].

\section{B. Demonstrator environment}

During 2017 and 2018, the novel CH-type cavity design has been tested and validated in two measurement campaigns, with $\mathrm{CHO}$ being the first of series to be extensively examined $[23,24,39]$. One of the main injectors at GSI, which delivers beam to the GSI UNILAC by default, was used to inject beam to the demonstrator test stand. Beam from the High Charge State Injector (HLI) (not to be confused with the High Current Injector (HSI [40])) has been delivered to $\mathrm{CHO}$ (installed in a test cryomodule) [41]. The IH-DTL as main part of the HLI is designed with the KOmbinierte NUll Grad Struktur (KONUS, in engl.: combined zero degree structure) beam dynamics concept $[42,43]$, which introduces a nonlinear transformation to the bunch shape in the longitudinal phase plane. This arises from using synchronous phases around $0^{\circ}$ in the most gaps instead of applying typical $-30^{\circ}$ constantly. For longitudinal and transversal matching of the beam to the demonstrator cryomodule, two rebunchers, two quadrupole duplets and a quadrupole triplet were available, as well as three beam steerers for alignment (see Fig. 1).
Phase probe sensors were used to determine the beam energy by time of flight (ToF) measurements. The beam profile and position are measured with secondary electron emission (SEM) grids. For longitudinal bunch shape measurements [44] a Feschenko monitor [45] was used. Within the demonstrator environment a low beam current was available, which makes space charge effects negligible.

\section{Principle of tomographic reconstruction}

The distribution $f(x, y)$ (see Fig. 2) has to be recalculated from a set of projections $f_{i}\left(x^{\prime}\right)$. The tomographic reconstruction method also appears in medical diagnostics, where it is used for body imaging. A wide range of reconstruction algorithms already exists for clinical purpose, but they are commonly formulated using linear mappings between $f(x, y)$ and $f_{i}\left(x^{\prime}\right)$ (see Fig. 2), or explicitly based on rotational transformations. For accelerator applications, it is unusual to alter the particle distribution in the phase space by a rigid rotation. Optical elements in the beam line are used to alter the bunch shape, which in most cases is characterized by shearing. When the bunch transformation can be expressed in this way, it is possible to preprocess the data into a sinogram to be used as input for common reconstruction algorithms. In some cases, the bunch transfer is not linear, therefore it is not useful to use sinograms [46]. Suitable algorithms have to be adjusted to this scenario. The algebraic reconstruction technique (ART) [47] and the maximum entropy tomography reconstruction (MENT) [48] already have been used for longitudinal phase space reconstruction $[48,49]$. As ART needs a high number of measurements, a different approach has been investigated for the HELIAC. The non-negative least squares method (NNLS) [50] is used to solve the reconstruction problem in the longitudinal phase plane.

\section{RECONSTRUCTION METHOD}

In order to derive the unknown bunch shape in the longitudinal phase space, different projections must be measured. Therefore, the two rebunchers were used to provide a variety of bunch shapes at the bunch shape monitor (BSM).

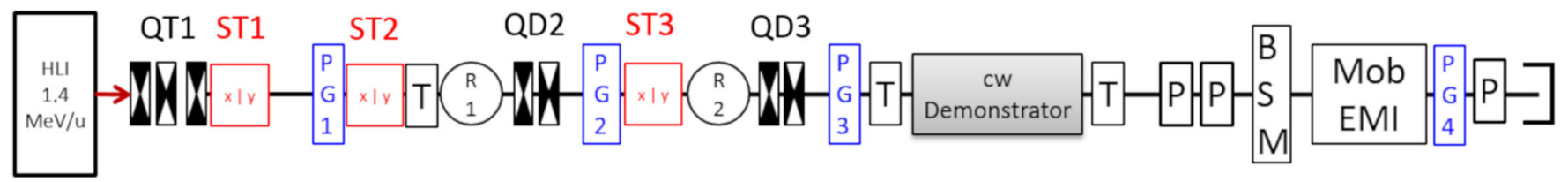

FIG. 1. Demonstrator Environment 2018. QT: Quadrupole Triplet, QD: Quadrupole Duplet, R: Rebuncher, xly: Beam Steerer, PG1-4: SEM-Grid, T: Beam Current Transformer, P: Phase Probe, BSM: Bunch Shape Monitor, EMI: Emittance Meter [23]. 

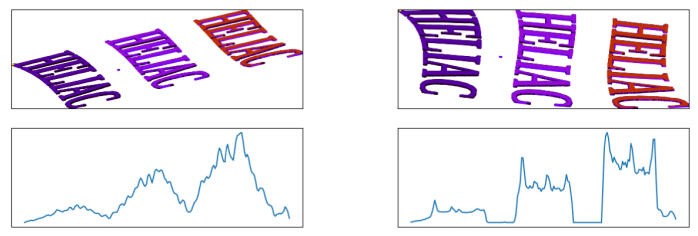

FIG. 2. Example of reconstruction setup for a transformation between image and trace. The observed traces constrain the shape of the reconstructed object [51].

\section{A. Bunch shape monitor}

The key device for the measurements is the Feschenko bunch shape monitor. It provides a longitudinal bunch density distribution for heavy ion beams and offers a high time/phase resolution of better $1 \mathrm{deg}$. at $108.408 \mathrm{MHz}$, as well as sufficient accuracy to o be used for the reconstruction method. The operational principle of the monitor is based on placing a metal wire into the beam line and recording the secondary electron emission caused by the beam-wire interaction. Additional electromagnetic lenses improve the signal to noise ratio significantly. A detailed description can be found in [45]. The monitor was located at the end of the line, behind the two rebunchers R1 and R2 as well as behind the cavity (see Fig. 1).

\section{B. Reconstruction}

For the reconstruction, the relation from the input coordinates at the beginning of the beam line (i.e., exit of the HLI) to the coordinates at the Feschenko Monitor must be known. The beamline was described by using the particle tracking code DYNAMION [52], which allows us to monitor individual particles along their trajectory. Disposing a grid as input distribution and tracking it through the beamline makes it possible to map two positions in the beam line as a function $\vec{f}_{i}\left(\vec{x}_{\text {in }}\right)$. The mapping is produced from the phase space at the injector HLI to the phase space at the BSM. The simulation of the beam line must be repeated for each buncher setting $i$. Additionally, the measurements can be described as $A_{i}\left(\phi, \vec{X}_{\text {in }}\right)$ for a set of input coordinates $\vec{X}_{\text {in }}$ (see Fig. 3). For a given particle of output phase $\phi$, different sets of input coordinates $\vec{x}_{\mathrm{in}, i}$ exist: $\vec{x}_{\mathrm{in}, i}(\phi)$ is ambiguous. For example, a slow particle with a high input phase and a fast particle with a low input phase both will be measured at the same phase in a distant point. With this effect, a infinite line in the input plane corresponds to one measured phase. A range of measured phases is represented by an area in the input plane. For different buncher settings, the area will have diverse orientations. By stacking up all back projections where a bunch signal was measured, we get an image where the bunch is present and where not, i.e., $N_{i}\left(\vec{x}_{\text {in }}\right)=\left|\left\{\phi_{i}\left(\vec{x}_{\text {in }}\right) \mid A\left(\phi_{i}\left(\vec{x}_{\text {in }}\right)\right) \neq 0\right\}\right|$. This is used to define the area, in which the detailed solver needs to be applied. Furthermore, the mapping from the input to the measurements $A_{i}\left(\phi, \vec{X}_{\text {in }}\right)$ is expressed in terms of a matrix

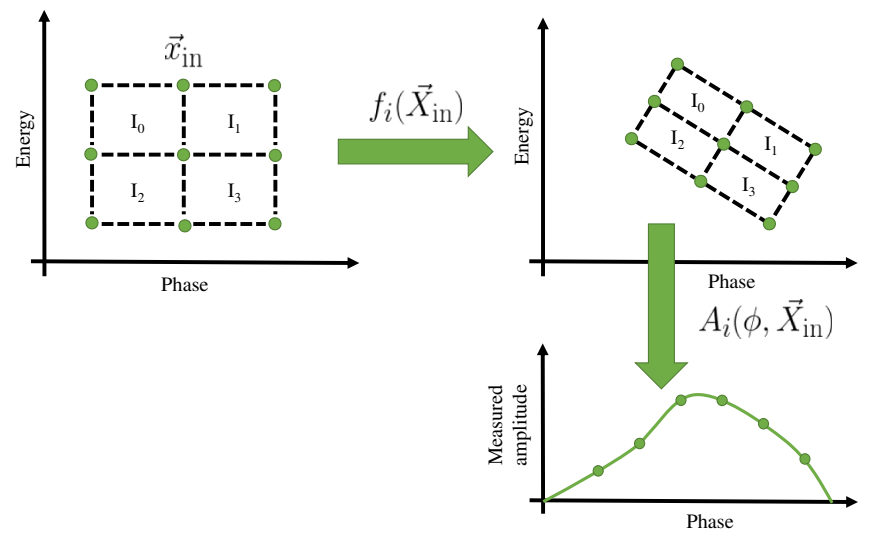

FIG. 3. The input distribution $\vec{X}_{\text {in }}$ is transported trough the beam line by $f_{i}\left(\vec{X}_{\text {in }}\right)$ and projected $A_{i}\left(\phi, \vec{X}_{\text {in }}\right)$.

multiplication $\overrightarrow{A_{i}}=B_{i} \cdot \vec{X}$, which is a discretized version of our mapping (The measurement data and the particle positions are discrete). With this discretization, also nonlinear mappings are expressed in terms of $B_{i}$, which is useful to determine the input distribution $\vec{X}$ with given measurements $\vec{A}_{i}$. Therefore, all mappings and all measurements are stacked up into one equation $\vec{A}=B \cdot \vec{X}$. The dimension of $B$ is therefore (Combined length of all measurements, number of input grid points). The standard least squared approach would yield negative particle densities. As this is nonphysical, a non-negative least squares approach is chosen, which restricts the result to be $\vec{X} \geq 0$ :

$$
\begin{gathered}
\text { minimize } f(\vec{X})=|B \vec{X}-\vec{A}| \\
\text { subject to } \quad \vec{X} \geq 0 .
\end{gathered}
$$

The mapping $B$ of the artificial input distribution $\vec{X}$ to all measured traces $\vec{A}$ should show minimal difference. As $\vec{A}$ is given by the measurements and $B$ is obtained by simulations, the only unknown is $\vec{X}$ and can be solved for, yielding the intensity at each discrete input position.

\section{RESULTS}

\section{A. Measurements}

All elements in the matching line were adjusted to a state with minimal beam loss for a wide range of rebuncher focusing strengths. A hundred of BSM-measurements have been conducted with $\mathrm{Ar}^{9+}$ beam from the HLI. Combinations of different rebuncher voltages $(R 1, R 2)$ were applied, providing for a transition from a defocused to an overfocused beam. Exemplary measurements are presented in Fig. 4. As shown as measured traces arranged as waterfall diagram, the bunch shape is asymmetric due to the KONUS beam dynamics of the HLI-IH-DTL. This makes the use of an advanced reconstruction technique 


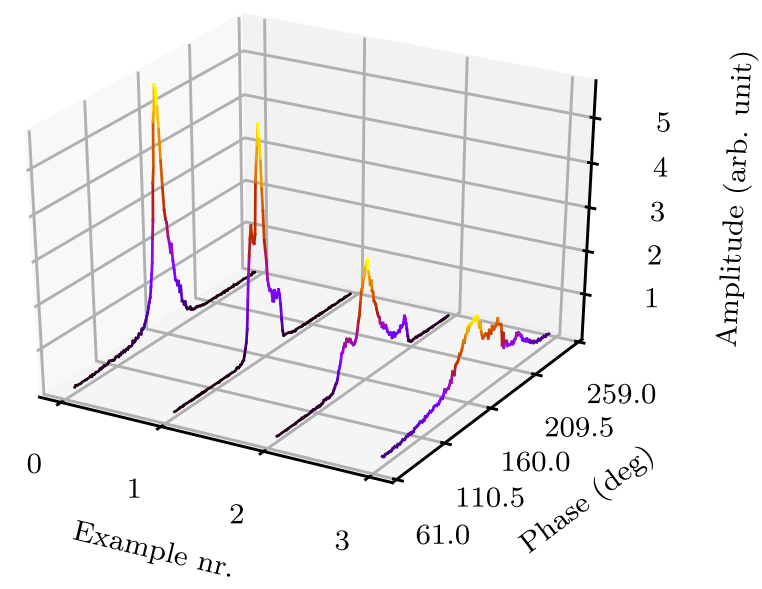

FIG. 4. Examples of the bunch shape measurements with the BSM for four different rebuncher settings (108 MHz). The color corresponds to the respective amplitude.

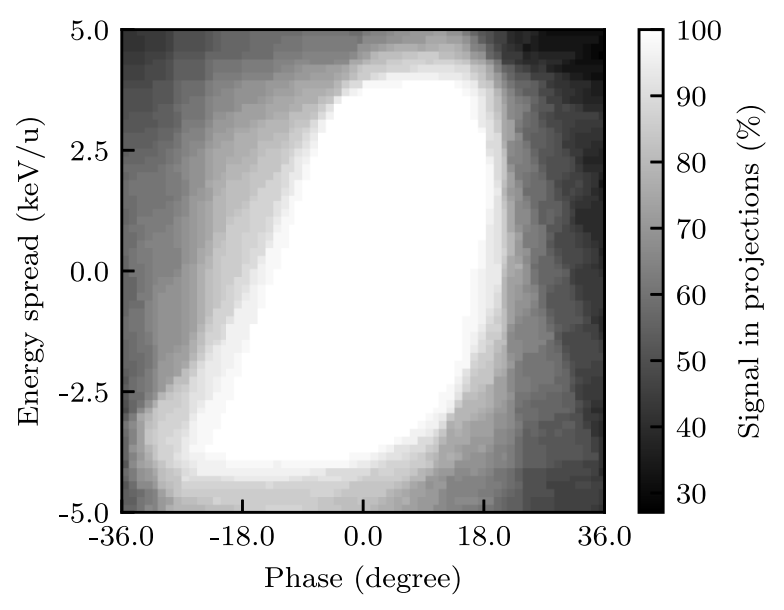

FIG. 5. By back projecting the traces onto the input plane, areas are marked in which no signal is present and vice versa. necessary, which also considers nonelliptical, arbitrary bunch shapes in the longitudinal phase plane. After detailed analysis of the experimental data, we decided to cut $6 \%$ of the signal in order to remove unwanted background. The traces were mutually realigned to their respective center of mass. In accordance to the algorithm the transmission was assumed to be $100 \%$. The BSM data is presented with the head of the bunch being displayed on the right side and the tail on the left (i.e., the beam is divergent). In the following, the phase planes are displayed using the same convention.

\section{B. Reconstruction}

As described in the previous section, the back projections of the bunch shape traces are used to determine the area of the bunch in the longitudinal phase space. These boundary values are used for the NNLS solver. This two step procedure enhances the analysis performance and increases the reliability by using two methods (backprojection and NNLS reconstruction). As the backprojections are based on data for different angles phi, they overlap in the reconstruction image and the parts of the BSM signal with $0 \%$ intensity are not processed. For the white area (100\% in Fig. 5), where all measurements hint the existence of the bunch, the RMS-emittance is evaluated as $\epsilon_{\mathrm{RMS}}=18 \mathrm{keV} / \mathrm{u} \mathrm{deg}$. This emittance is in good agreement with former simulations of the HLI, which yielded in an RMS emittance of $13.5 \mathrm{keV} / \mathrm{u}$ deg [53]. The reconstructed emittance is slightly increased, as the shape reconstruction does not take the signal strength into account. By using the limits shown in Fig. 5, the NNLS solver was applied to reconstruct the exact bunch shape and density distribution with the derived boundary values. The NNLS solver reveals a more complicated shape (see Fig. 6). The RMS emittance is $\epsilon_{\mathrm{RMS}}=27.3 \mathrm{keV} / \mathrm{u} \mathrm{deg}$. In the center of the distribution, a remarkably small spot with high intensity is recognizable. The analysis of the reconstructed
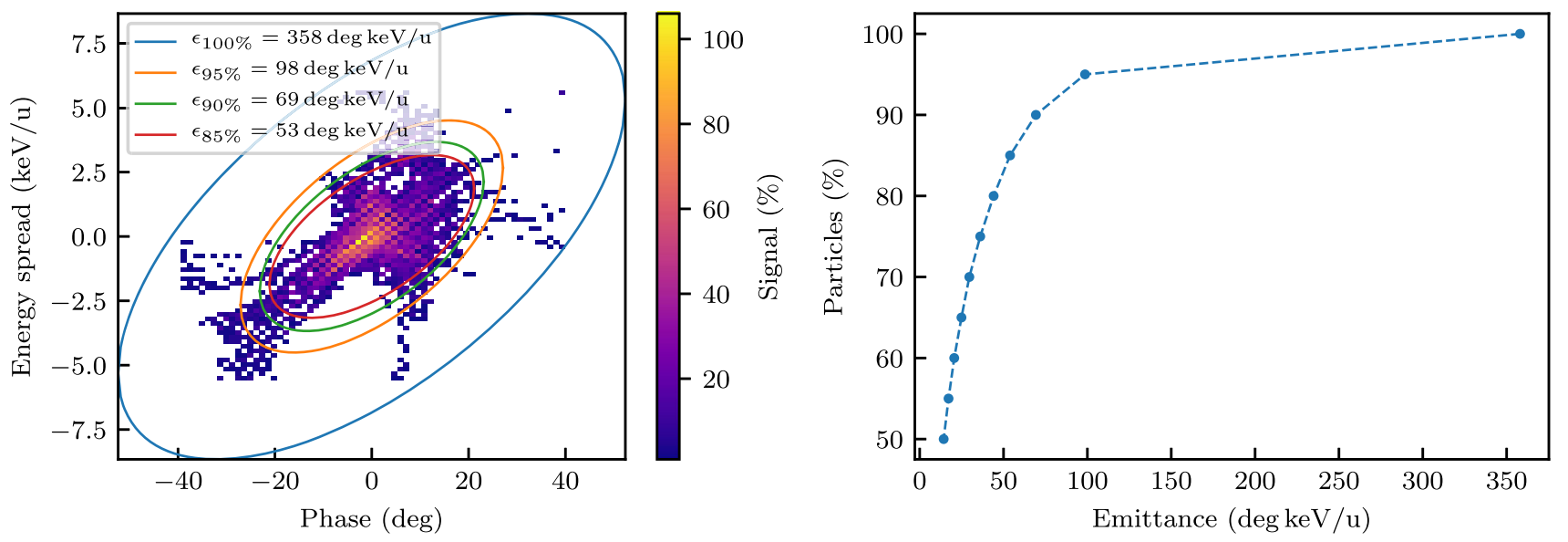

FIG. 6. Brilliance analysis of the reconstructed distribution. (a) Samples of the fitted ellipses around a fraction of the density distribution. (b) Relation of the emittance on the fraction of particles. 


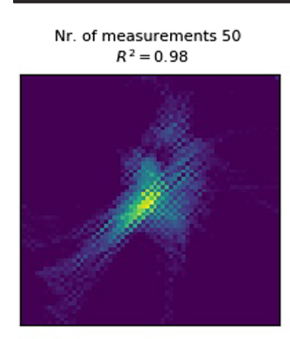

Nr. of measurements 20 $R^{2}=0.98$

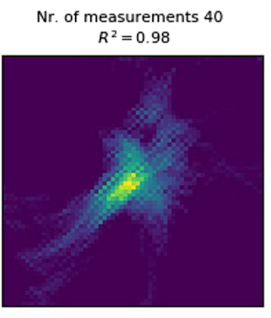

Nr. of measurements 10

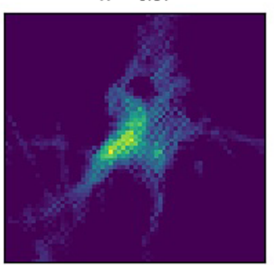

Nr. of measurements 30
$R^{2}=0.98$

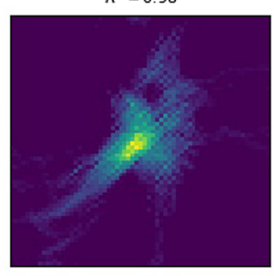

Nr. of measurements 5 $R^{2}=0.89$

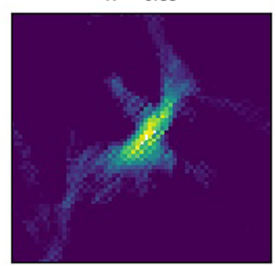

FIG. 7. The number of measurements used for the reconstruction is reduced, but the reconstructed shape stays equivalent.

emittance is also presented in Fig. 6. Selected ellipses surrounding a fraction of the particles are displayed. The observed emittance of the bunch is dominated by a marginal percentage of the particles $(5 \%$ of the particle distribution), while most of the particles are concentrated in the center. The reconstructed emittance fits well with the original assumptions for the design of the accelerator. Recently, the BSM was mounted and commissioned at a different position directly at the exit of the injector HLI (i.e., at the reconstruction point) and a dedicated measurement campaign is foreseen to confirm the reconstruction result.

\section{Low projection count}

A view of the longitudinal phase space makes machine optimization more efficient for operators, the reconstruction should be conducted as fast as possible. For this purpose, a high number of measurements is counterproductive. Performing the reconstruction with less projections returns a stable view of the phase space. With 10 measurements as input for the solver a compromise between performance and accuracy can be made. (see Fig. 7). The selection of the subset of measurements must be done carefully. The corresponding rebuncher settings should not be similar, they should reflect the variety of the full set for optimal results. The coefficient of determination $R^{2}$, a regression score which is defined by the measurements $y_{i}$ and the fitted data $\hat{y}_{i}$ as

$$
R^{2}=1-\frac{\sum\left(y_{i}-\hat{y}_{i}\right)^{2}}{\sum\left(y_{i}-\bar{y}\right)^{2}},
$$

is acceptable high for 10 measurements as input, indicating that 10 measurements are enough to perform the reconstruction with a negligible loss of accuracy from $R^{2}(50$ measurements $)=0.98$ to $R^{2}(10$ measurements $)=$ 0.97. In the following the regression score is always calculated with $y$ containing all measured data, even if $\hat{y}$ is derived from a subset of the measurements. This investigation validates the reconstruction method, as from a low number of inputs to the solver we obtain results which are similar to those using a high number of measurements. The results are consistent.

\section{Validation: Cavity phase scan}

A set of measurements has been carried out to provide an additional validation of our reconstruction method. The previously unused cavity $\mathrm{CH} 0$ was used to accelerate the bunch at a cavity voltage of $U_{0}=3.3 \mathrm{MV}$. The rf phase of the cavity was varied. The amplitudes of the rebuncher cavities were fixed to match the beam energy and orientation to the cavity. By varying the cavity rf phase,
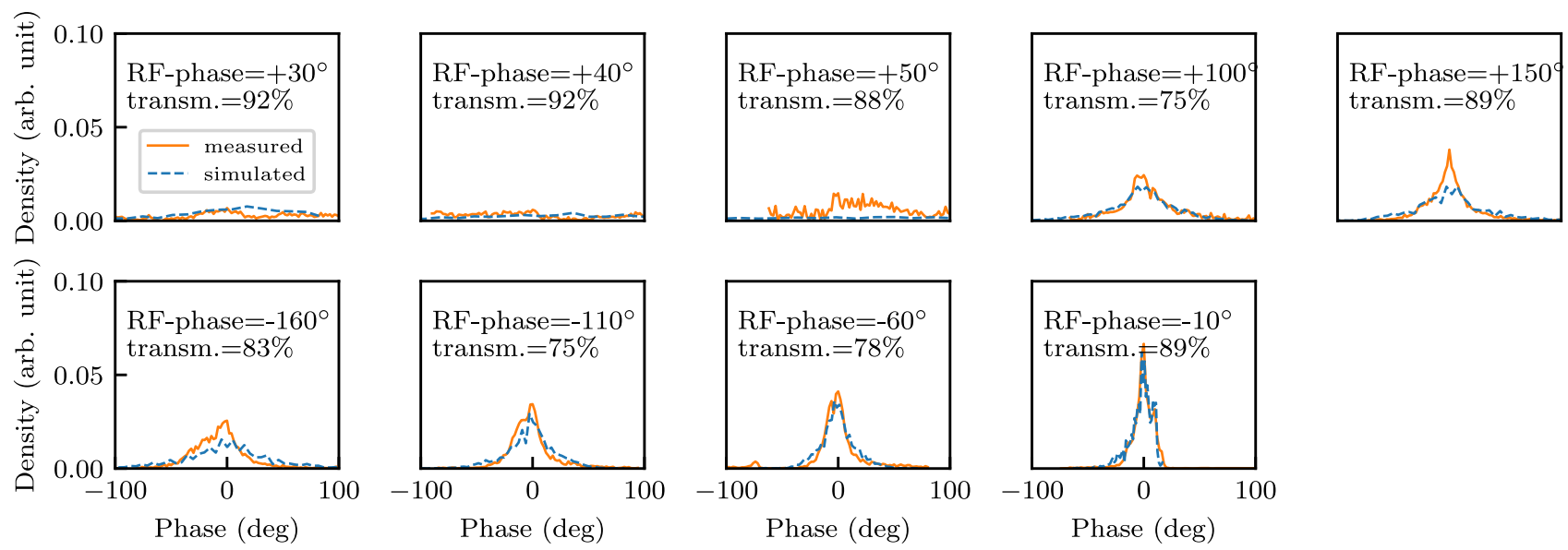

FIG. 8. The phase of the cavity $\mathrm{CHO}$ is varied at fixed rebuncher amplitude. This phase scan is reproduced with DYNAMION simulations using the reconstructed bunch shape as input distribution. The cavity gradient is $U_{0}=3.3 \mathrm{MV}$. The maximum acceleration gain is reached for a rf-phase $0^{\circ}$. 
different measurements at the Feschenko monitor have been taken. A beam dynamics simulation of the reconstructed bunch must be able to reproduce these measurement results as well. From Fig. 8 it follows that the measurements show a wide range from a peaked distribution to a flat one. The flat distributions are caused by the injection of the bunch at the boundary of the separatrix, which practically tears the bunch apart. The simulations and measurements are in good coincidence with a coefficient of determination $R^{2}=0.79$, even though one outlier is measured at $130^{\circ}$. For the measurements with the $0 \leq \mathrm{rf}-$ phase $\leq 30^{\circ}$, the measured signal is weak and noisy, as it is expected for a completely defocused bunch. The reduced $R^{2}$ comparing to the results from the rebuncher scans with $R^{2}=0.98$ is most likely caused by particle losses, thus a lower transmission, which is linked to the transverse size of the beam and misalignment of the cavity, which was not considered for these simulations.

\section{CONCLUSION AND OUTLOOK}

Sufficient experimental data has been collected to reconstruct the longitudinal phase space characteristics of a bunched ion beam. The shape of the bunch and its density distribution are reconstructed with the NNLS algorithm from the results of the beam dynamics code DYNAMION. This is based on a variety of bunch shape measurements at the end of the beam-line under different settings of cavity parameters. Independent measurements have been carried out to further verify our findings. As of now, the matching of the beam to the superconducting continuous wave demonstrator and to the HELIAC can be studied with a higher level of detail to aim for advanced performance of the entire system. It is intended to further validate the obtained results by comparing the reconstructed with the measured longitudinal projection at the exit of the HLI, i.e., at the reconstruction point. For the design beam current of $1 \mathrm{~mA}$, additional space charge considerations must be discussed in prospective measurements.

[1] M. Block et al., Direct mass measurements above uranium bridge the gap to the island of stability, Nature (London) 463, 785 (2010).

[2] J. Khuyagbaatar et al., ${ }^{48} \mathrm{Ca}+{ }^{249} \mathrm{Bk}$ Fusion Reaction Leading to Element $Z=117$ : Long-lived $\alpha$-Decaying ${ }^{270} \mathrm{Db}$ and Discovery of ${ }^{266} \mathrm{Lr}$, Phys. Rev. Lett. 112, 172501 (2014).

[3] W. Barth, W. Bayer, L. Dahl, L. Groening, S. Richter, and S. Yaramyshev, Upgrade program of the high current heavy ion unilac as an injector for FAIR, Nucl. Instrum. Methods Phys. Res., Sect. A 577, 211 (2007).

[4] W. Barth et al., $\mathrm{U}^{28+}$-intensity record applying a $\mathrm{H}_{2}$-gas stripper cell, Phys. Rev. Accel. Beams 18, 040101 (2015).
[5] A. Adonin and R. Hollinger, Beam brilliance investigation of high current ion beams at GSI heavy ion accelerator facility, Rev. Sci. Instrum. 85, 02 A727 (2014).

[6] S. Yaramyshev et al., Virtual charge state separator as an advanced tool coupling measurements and simulations, Phys. Rev. Accel. Beams 18, 050103 (2015).

[7] W. Barth et al., High brilliance uranium beams for the GSI FAIR, Phys. Rev. Accel. Beams 20, 050101 (2017).

[8] L. Groening et al., Benchmarking of measurement and simulation of transverse rms-emittance growth, Phys. Rev. Accel. Beams 11, 094201 (2008).

[9] W. Barth et al., A superconducting CW-linac for heavy ion acceleration at GSIX, EPJ Web Conf. 138, 01026 (2017).

[10] W. Barth et al., Superconducting CH-cavity heavy ion beam testing at GSI, J. Phys. Conf. Ser. 1067, 052007 (2018).

[11] F. Dziuba et al., First cold tests of the superconducting CW demonstrator at GSI, in Proceedings of RuPAC'16, St. Petersburg, Russia (2017), pp. 83-85, http://jacow.org/ rupac2016/papers/wecbmh01.pdf.

[12] H. Podlech, U. Ratzinger, H. Klein, C. Commenda, H. Liebermann, and A. Sauer, Superconducting CH structure, Phys. Rev. Accel. Beams 10, 080101 (2007).

[13] M. Schwarz et al., Beam dynamics simulations for the new superconducting CW heavy ion linac at GSI, J. Phys. Conf. Ser. 1067, 052006 (2018).

[14] M. Gusarova, W. A. Barth, S. Yaramyshev, M. Miski-Oglu, M. Basten, and M. Busch, Design of the two-gap superconducting re-buncher, J. Phys. Conf. Ser. 1067, 082005 (2018).

[15] K. Taletskiy, M. Gusarova, W. A. Barth, M. Basten, M. Busch, M. Miski-Oglu, and S. Yaramyshev, Comparative study of low beta multi-gap superconducting bunchers, J. Phys. Conf. Ser. 1067, 082006 (2018).

[16] A. E. Aksent'ev et al., Modeling of proton beam dynamics in an accelerator-driver at $600-1000 \mathrm{MeV}$ and investigation of the electrodynamic characteristics of accelerating cavities, At. Energ. 117, 347 (2015).

[17] S. Polozov and A. Fertman, High-energy proton beam accelerators for subcritical nuclear reactors, At. Energ. 113, 192 (2013).

[18] Z. Wang et al., Beam commissioning for a superconducting proton linac, Phys. Rev. Accel. Beams 19, 120101 (2016).

[19] I. Mardor et al., The Soreq Applied Research Accelerator Facility (SARAF): Overview, research programs and future plans, Eur. Phys. J. A 54, 91 (2018).

[20] L. V. Grigorenko et al., Scientific program of DERICAprospective accelerator and storage ring facility for radioactive ion beam research, Phys. Usp. 62, 675 (2019).

[21] R. Laxdal, K. Fong, M. Laverty, A. Mitra, R. Poirier, I. Sekachev, and V. Zvyagintsev, Recent progress in the superconducting RF program at TRIUMF/ISAC, Physica C (Amsterdam) 441, 13 (2006).

[22] P. Ostroumov and F. Gerigk, Superconducting hadron linacs, Rev. Accel. Sci. Techol. 06, 171 (2013).

[23] W. Barth et al., First heavy ion beam tests with a superconducting multigap $\mathrm{CH}$ cavity, Phys. Rev. Accel. Beams 21, 020102 (2018). 
[24] S. Minaev, U. Ratzinger, H. Podlech, M. Busch, and W. Barth, Superconducting, energy variable heavy ion linac with constant $\beta$, multicell cavities of CH-type, Phys. Rev. Accel. Beams 12, 120101 (2009).

[25] F. Dziuba et al., Development of superconducting crossbar-H-mode cavities for proton and ion accelerators, Phys. Rev. Accel. Beams 13, 041302 (2010).

[26] G. Clemente, U. Ratzinger, H. Podlech, L. Groening, R. Brodhage, and W. Barth, Development of room temperature crossbar-H-mode cavities for proton and ion acceleration in the low to medium beta range, Phys. Rev. Accel. Beams 14, 110101 (2011).

[27] M. Schwarz et al., Reference beam dynamics layout for the SC CW heavy ion HELIAC at GSI, Nucl. Instrum. Methods Phys. Res., Sect. A 951, 163044 (2020).

[28] M. Yarmohammadi Satri, A. M. Lombardi, and F. Zimmermann, Multiobjective genetic algorithm approach to optimize beam matching and beam transport in highintensity hadron linacs, Phys. Rev. Accel. Beams 22, 054201 (2019).

[29] O. Brunner et al., Assessment of the basic parameters of the CERN superconducting proton linac, Phys. Rev. Accel. Beams 12, 070402 (2009).

[30] V. Palmieri et al., Installation in the LNL ALPI linac of the first cryostat with four niobium quarter wave resonators, Nucl. Instrum. Methods Phys. Res., Sect. A 382, 112 (1996).

[31] B. Walasek-Hoehne, C. Andre, P. Forck, E. Gutlich, G. Kube, P. Lecoq, and A. Reiter, Scintillating screen applications in accelerator beam diagnostics, IEEE Trans. Nucl. Sci. 59, 2307 (2012).

[32] T. Giacomini, S. Barabin, P. Forck, D. Liakin, and V. Skachkov, Development of residual gas profile monitors at GSI, AIP Conf. Proc. 732, 286 (2004).

[33] U. Ratzinger et al., The $70 \mathrm{MeV}$ p-injector design for FAIR, AIP Conf. Proc. 773, 249 (2005).

[34] W. Barth, A. Adonin, S. Appel, P. Gerhard, M. Heilmann, F. Heymach, R. Hollinger, W. Vinzenz, H. Vormann, and S. Yaramyshev, Heavy ion linac as a high current proton beam injector, Phys. Rev. Accel. Beams 18, 050102 (2015).

[35] A. Adonin, W. Barth, F. Heymach, R. Hollinger, H. Vormann, and A. Yakushev, Production of high current proton beams using complex H-rich molecules at GSI, Rev. Sci. Instrum. 87, 02 B709 (2016).

[36] P. Forck, Minimal invasive beam profile monitors for high intense hadron beams, in Proceedings of the International Particle Accelerator Conference, Kyoto, Japan (ICR, Kyoto, 2010), pp. 1261-1265, https://www.researchgate .net/publication/266247467_Minimal_invasive_beam_ profile_monitors_for_high_intense_hadron_beams.

[37] F. Herfurth et al., The HITRAP facility for slow highly charged ions, Phys. Scripta T166, 014065 (2015).

[38] S. Busold et al., Shaping laser accelerated ions for future applications the LIGHT collaboration, Nucl. Instrum. Methods Phys. Res., Sect. A 740, 94 (2014).

[39] S. Yaramyshev et al., Advanced approach for beam matching along the multi-cavity SC CW linac at GSI, J. Phys. Conf. Ser. 1067, 052005 (2018).
[40] L. Groening et al., Experimental evidence of space charge driven emittance coupling in high intensity linear accelerators, Phys. Rev. Lett. 103, 224801 (2009).

[41] P. Gerhard et al., Commissioning of a new CW radio frequency quadrupole at GSI, in Proceedings of the International Particle Accelerator Conference, Kyoto, Japan (ICR, Kyoto, 2010), p. 741743, http://accelconf .web.cern.ch/AccelConf/IPAC10/papers/mopd028.pdf.

[42] R. Tiede, G. Clemente, H. Podlech, U. Ratzinger, A. C. Sauer, and S. Minaev, LORASR code development, in Proceedings of the 10th European Particle Accelerator Conference, Edinburgh, Scotland, 2006 (EPS-AG, Edinburgh, Scotland, 2006), Vol. C060626, pp. 2194-2196, https://www .researchgate.net/publication/239615516_LORASR_code_ development.

[43] R. Tiede, U. Ratzinger, H. Podlech, C. Zhang, and G. Clemente, KONUS beam dynamics designs using H-mode cavities, in Proc. HB'08, TN, USA (2008), pp. 223-230, https://www.researchgate.net/publication/234035056_ KONUS_eam_dynamics_designs_using_H-mode_cavities.

[44] T. Sieber et al., Bunch shape measurements at the GSI CWlinac prototype, in Proc. IPAC'18 (2018), pp. 2091-2094, http://jacow.org/ipac2018/papers/wepak006.pdf.

[45] A. Feschenko, Methods and instrumentation for bunch shape measurements, in Proceedings of the 19th Particle Accelerator Conference, Chicago, IL, 2001 (IEEE, Piscataway, NJ, 2001), pp. 517-521, https://ieeexplore.ieee.org/ document/987557.

[46] J.-B. Lallement et al., Linac4 Transverse and Longitudinal Emittance Reconstruction in the Presence of Space Charge, in Proc. LINAC'14 (2014), pp. 913-915, https://www .researchgate.net/publication/273256768_Linac4_ transverse_and_longitudinal_emittance_reconstruction_ in_the_presence_of_space_charge.

[47] R. Gordon, R. Bender, and G. Herman, Algebraic reconstruction technique (ART) for three-dimensional electron microscopy and x-ray photography, J. Theor. Biol. 29, 471 (1970).

[48] K. Hock, M. G. Ibison, D. J. Holder, B. D. Muratori, A. Wolski, G. Kourkafas, and B. J. A. Shepherd, Beam tomography research at Daresbury laboratory, Nucl. Instrum. Methods Phys. Res., Sect. A 753, 38 (2014).

[49] D. Malyutin, M. Gross, I. Isaev, M. Khojoyan, G. Kourkafas, M. Krasilnikov, B. Marchetti, M. Otevrel, F. Stephan, and G. Vashchenko, Longitudinal phase space tomography using a booster cavity at PITZ, Nucl. Instrum. Methods Phys. Res., Sect. A 871, 105 (2017).

[50] C. L. Lawson and R. J. Hanson, Solving Least Squares Problems (Society for Industrial and Applied Mathematics, Philadelphia, PA, USA, 1987).

[51] S. Lauber et al., Reconstruction of the longitudinal phase portrait for the SC CW heavy ion HELIAC at GSI, in Proc. IPAC'19 (2019), pp. 898-901, https://doi.org/10.18429/ JACoW-IPAC2019-MOPTS024.

[52] S. Yaramyshev, W. Barth, L. Groening, A. Kolomiets, and T. Tretyakova, Development of the versatile multi-particle code DYNAmion, Nucl. Instrum. Methods Phys. Res., Sect. A 558, 90 (2006).

[53] U. Ratzinger, Effiziente Hochfrequenz-Linearbeschleuniger für leichte und schwere Ionen (Phys. Dept., Goethe University, Frankfurt am Main, Germany, 1998). 